\title{
On Parametric Pole-Placement for a Class of Linear Systems
}

Jeng Yih Juang

Professor, Department of Merchant Marine, National Taiwan Ocean University, Keelung, Taiwan R. O. C., juang@sun21.ntou.edu.tw

Follow this and additional works at: https://jmstt.ntou.edu.tw/journal

Part of the Engineering Commons

\section{Recommended Citation}

Juang, Jeng Yih (1997) "On Parametric Pole-Placement for a Class of Linear Systems," Journal of Marine Science and Technology. Vol. 5: Iss. 1, Article 1.

DOI: $10.51400 / 2709-6998.2532$

Available at: https://jmstt.ntou.edu.tw/journal/vol5/iss1/1

This Research Article is brought to you for free and open access by Journal of Marine Science and Technology. It has been accepted for inclusion in Journal of Marine Science and Technology by an authorized editor of Journal of Marine Science and Technology. 


\section{On Parametric Pole-Placement for a Class of Linear Systems}

Acknowledgements

The author is grateful to the National Science Counsil, R.O.C., for partial financial support under contract no. NSC-86-2213-E-019-004.

This research article is available in Journal of Marine Science and Technology: https://jmstt.ntou.edu.tw/journal/vol5/ 


\title{
ON PARAMETRIC POLE-PLACEMENT FOR A CLASS OF LINEAR SYSTEMS
}

\author{
Jeng Yih Juang*
}

Keywords: Parametrization, Pole-placement, Multivariable systems.

\begin{abstract}
This paper presents an innovative approach for parametrizing a class of state-feedback pole-placement controllers through the minimum number of effective free parameters. The approach is based on the theory of hypothetical loop-decoupling. Linear and nonlinear functions of parameter coupling in the feedback gain matrices are disclosed to the problem of generic parametric poleplacement for a linear time-invariant controllable system. With the class depicted by the controllability indices for the hypothetical control loops, parametrization for state-feedback design can be achieved. The flexibility in loop pole-assignment for a multi-input system also affects the choice for the gain matrix. The excellence of the proposed method is illustrated by several numerical examples.
\end{abstract}

\section{INTRODUCTION}

In the theory of modern control system design, pole-placement (also known as pole-assignment, or eigenvalue assignment) is one of most fundamental disciplines. In the time-domain for an MIMO controllable system, this task is achieved by a linear state variable feedback (LSVF) law. It is well-known that the solution to the problem is not unique, and there exists degree of freedom in the gain matrix to be used so as to improve other performances of control purposes.

The possible applications can be found to be stabilization, hypothetical loop-decoupling [1], output decoupling [2], linear quadratic regulator (LQR) and optimal control, asymptotic observer design, robust control and deadbeat control [3]. Minimum-time deadbeat control (MTDC) and minimum-time minimum-gain deadbeat control (MTMGDC) design for a

Paper Received January, 1997. Revised April, 1997. Accepted April, 1997. Author for Correspondence: Jeng Yih Juang.

*Professor, Department of Merchant Marine, National Taiwan Ocean University,Keelung,Taiwan R.O.C.E_mail:juang@sun21.ntou.edu.tw reachable linear discrete-time system, in our opinion, belong to the generic pole-placement problem.

Since 1980 , the research topic on parametric state feedback design for a multi-input linear system has been receiving great attentions in dealing with multivariable optimal control in the time-domain, c.f. Roppenecker [4] and the references cited therein. Robust pole assignment design utilizing the degrees of freedom in LSVF was suggested by Kautsky et. al. [5], Dickman [6] and other authors to follow. Fahmy and O'Reilly [7], O'Reilly and Fahmy [8] considered the problem of eigenvalue assignment (EA) with the framework of parametric eigenstructure assignment. The non-uniqueness of the set of assignable eigenvectors reflects the design degrees of freedom. The suggested number of classes of EA controllers is equal to the number of admissible Jordan forms of the closed-loop systems. They gave the result of minimum parametrization through iterating process of reducing the number of Jordan blocks as long as making some block as longest as possible. However, the prescribed closed-loop eigenvalues are not allowed to include the open-loop eigenvalues, though this limitation was further extended.

As the work on MTDC topics, Sebakhy and Abdel-Moneim [9] tackled the problem of shaping the transient response from optimal control. This opens the research line on the topic of MTDC parametrization. Schlegel [10] considered the MTDC problem based on the fact of a class of controllers that makes $A_{c}$ similar to $m$ nilpotent Jordan blocks, ( $m$ : the number of inputs) with the largest block of length $\mu$, the controllability index, as the way Kucera [11] did. He also pointed out that the minimum parametrzation formula of Sebakhy [9] was incorrect, and gave an explicit parametrization result through the minimum number of $N_{p}=m n-\mu(1)-3 \mu(2)-\ldots-(2 m-1) \mu(m)$ effective real parameters for the class of controllers that solves MTDC, (here, $n$ the number of states, $\mu(i)$ 's are controllability indices arranged with $\mu(i) \geq$ $\mu(i+1))$. 
Amin [12] also attacked the problem of MTDC parametrization from EA based on similarity of the system matrix to nilpotent Jordan blocks. Elabdalla and Amin [13], and Amin [14] tackled the MTDC problem based on the decoupling theory of Falb and Wolovich [2], and get the minimum prarmetrization formula as the one obtained by Schlegel [10]. Amin [15] also attacked the problem of minimum-time output deadbeat control (MTODC) based on said decoupling theory and the properties of invertible systems, recently.

Our objective, in this paper, is to present an innovative approach based on hypothetical loopdecoupling theory for parametrizing a class of statefeedback pole-placement controllers through the minimum number of effective free parameters. The class of control design is depicted by the choice of the set of controllability indices under the framework of the theory. The contribution of the paper is the explicit linear or nonlinear parametric form for the gain matrix representing the class of controllers for a generic pole-placement design. Linear and nonlinear functions of parameter coupling in the feedback gain matrices are disclosed to the problem of generic parametric pole-placement for a linear time-invariant controllable system. The flexibility in loop pole-assignment for a multi-input system also affects the choice for the gain matrix. Over-parametrization for the pole-placement of equal characteristic polynomial in control loops may result in that the extra parameters be not effective, this was illustrated by examples. Feedback gain matrix parametrization is believed to be helpful in the optimal control, e.g., robust pole-placement design, where parameters may further be tuned for other purpose. This achieves mixed pole-placement and optimal performance design works.

\section{MOTIVATION EXAMPLES}

\section{Naive Method}

Consider the following system,

$$
\dot{\mathrm{x}}(t)=\left[\begin{array}{cc}
-1 & 0 \\
0 & -1
\end{array}\right] \mathbf{x}(t)+\left[\begin{array}{ll}
1 & 0 \\
0 & 1
\end{array}\right] \mathbf{u}(t) .
$$

It is required to place closed-loop poles to $\{-2,-3\}$ by an LSVF law $\mathbf{u}(\mathrm{t})=\mathbf{F v}(\mathrm{t})-\mathbf{K x}(\mathrm{t})$. Let the feedback gain matrix be

$$
\mathbf{K}=\left[\begin{array}{ll}
a & b \\
c & d
\end{array}\right],(a, b, c, d) \in \Re
$$

Therefore, the characteristic equation for the closedloop system must be

$$
\left|\begin{array}{cl}
s+1+a b b \\
c \quad s+1+d
\end{array}\right| \begin{aligned}
& =s^{2}+(a+d+2) s+(a d+a+d+1-b c) \\
& =s^{2}+5 s+6,
\end{aligned}
$$

accordingly we have two constraints as follows:

$$
a+d=3 \text {, }
$$

and

$$
a d=2+b c \text {. }
$$

It seems that there left for us two degrees of freedom in $\mathbf{K}$. Actually, this is not exactly true. For $a$, $d$ to be real, the following constraint has to be met, too:

$$
b c \leq \frac{1}{4} .
$$

Again, one asks if there is only one degree of freedom left in $\mathbf{K}$ still, and if it is also an effective one. This motivates our research work on pole-placement with parametric state feedback.

Consider the Frobenius norm of $\mathbf{K}$, along with

$$
\begin{aligned}
\|\mathbf{K}\|_{F}^{2} & =a^{2}+d^{2}+b^{2}+c^{2} \\
& =(b-c)^{2}+5, \text { by Eqs. (1) and (2). }
\end{aligned}
$$

Clearly at $b=c,\|\mathbf{K}\|_{F}$ takes its minimum value of $\sqrt{5}$ hence the solution

$$
\mathbf{K}=\left[\begin{array}{cc}
\frac{3}{2} & \pm \frac{1}{2} \\
\pm \frac{1}{2} & \frac{3}{2}
\end{array}\right]
$$

becomes a normal matrix, and Robust pole-placement [6] can be achieved.

\section{Hypothetical Loop-decoupling Method [1]}

For this controllable system, the controllability indices [6] are $\mu_{1}=\mu_{2}=1$, the hypothetical outputs are defined as, $\theta_{1}(t)=\left[\begin{array}{ll}1 & \alpha\end{array}\right] \mathbf{x}(t), \theta_{2}(t)=\left[\begin{array}{ll}\delta & 1\end{array}\right] \mathbf{x}(t)$. (two free parameters: $\alpha, \delta$ ). Now observing the phase variables of the outputs as follows, $(s:=d / d t)$

$$
\begin{aligned}
& \theta_{1}(t)=\left[\begin{array}{ll}
1 & \alpha
\end{array}\right] \mathbf{x}(t), \\
& s \theta_{1}(t)=\left[\begin{array}{ll}
-1 & -\alpha
\end{array}\right] \mathbf{x}(t)+\left[\begin{array}{ll}
1 & \alpha
\end{array}\right] \mathbf{u}(t), \\
& \theta_{2}(t)=\left[\begin{array}{ll}
\delta & 1
\end{array}\right] \mathbf{x}(t), \\
& s \theta_{1}(t)=\left[\begin{array}{ll}
-\delta & -1
\end{array}\right] \mathbf{x}(t)+\left[\begin{array}{ll}
\delta & 1
\end{array}\right] \mathbf{u}(t)
\end{aligned}
$$

and consider two cases for loop assignments as (a) characteristic polynomial $\pi_{1}(s)=s+2, \pi_{2}(s)=s+3$. Put in 


$$
\begin{aligned}
& s \theta_{1}+2 \theta_{1}=\left[\begin{array}{ll}
1 & \alpha
\end{array}\right] \mathbf{x}(t)+\left[\begin{array}{ll}
1 & \alpha
\end{array}\right] \mathbf{u}(t):=\mathbf{v}_{1}, \\
& s \theta_{2}+3 \theta_{2}=\left[\begin{array}{ll}
2 \delta & 2
\end{array}\right] \mathbf{x}(t)+\left[\begin{array}{ll}
\delta & 1
\end{array}\right] \mathbf{u}(t):=\mathbf{v}_{2},
\end{aligned}
$$

The LSVF law for pole-placement is,

$$
\begin{aligned}
\mathbf{u}(t) & =\frac{1}{1-\delta \alpha}\left[\begin{array}{cc}
1 & -\alpha \\
-\delta & 1
\end{array}\right] \mathbf{v}(t) \\
& -\frac{1}{1-\delta \alpha}\left[\begin{array}{cc}
1-2 \delta \alpha & -\alpha \\
\delta & -\delta \alpha
\end{array}\right] \mathbf{x}(t),(\delta \alpha \neq 1)
\end{aligned}
$$

and feedback gain matrix is

$$
\mathbf{K}_{a}=\left[\begin{array}{cc}
1-\frac{\delta \alpha}{1-\delta \alpha} & -\frac{\alpha}{1-\delta \alpha} \\
\frac{\delta}{1-\delta \alpha} & 2+\frac{\delta \alpha}{1-\delta \alpha}
\end{array}\right], \delta \alpha \neq 1
$$

This leads to parameters coupling in nonlinear form. However, the number of degrees must be less than 2, for $\delta \alpha \neq 1$ has to be met. Particularly when we consider the following one-parameter solution for $\mathbf{K}_{a}$ :

$$
\begin{array}{ll}
\alpha=0, & \mathbf{K}_{a}=\left[\begin{array}{ll}
1 & 0 \\
\delta & 2
\end{array}\right], \\
\delta=0, & \mathbf{K}_{a}=\left[\begin{array}{cc}
1 & -\alpha \\
0 & 2
\end{array}\right] .
\end{array}
$$

This leads to linear form for the parametrization for $\mathbf{K}_{a}$. Accordingly, it is seen that at $\alpha=0$ or $\delta=0,\left\|\mathbf{K}_{a}\right\|_{F}$ attains its minimum of $\sqrt{5}$ too. This also clarify the result obtained by Eq. (4).

(b) characteristic polynomial $\pi_{1}(s)=s+3, \pi_{2}(s)=s+2$. Following similar hypothetical loop-decoupling procedures as Eqs (6) and (7), one gets the following feedback gain matrix:

$$
\mathbf{K}_{b}=\frac{1}{1-\delta \alpha}\left[\begin{array}{cc}
2-\delta \alpha & \alpha \\
-\delta & 1-2 \delta \alpha
\end{array}\right],(\delta \alpha \neq 1)
$$

for the second case. It is also seen that, the oneparameter solution for $\mathbf{K}_{a}$ is

$$
\begin{array}{ll}
\alpha=0, & \mathbf{K}_{b}=\left[\begin{array}{cc}
2 & 0 \\
-\delta & 1
\end{array}\right], \\
\delta=0, & \mathbf{K}_{b}=\left[\begin{array}{ll}
2 & \alpha \\
0 & 1
\end{array}\right] .
\end{array}
$$

Clearly at $\alpha=0$ or $\delta=0,\left\|\mathbf{K}_{b}\right\|_{F}$ attains its minimum of $\sqrt{5}$ as $\left\|\mathbf{K}_{a}\right\|_{F}$ does.

Return to Eq. (8), for the case of nonlinear coupling with two parameters again, consider

$$
\begin{aligned}
\mid \mathbf{K}_{a} \|_{F}^{2} & =\left(\frac{1}{1-\delta \alpha}\right)^{2}\left[(1-2 \delta \alpha)^{2}+\alpha^{2}+\delta^{2}+(2-\delta \alpha)^{2}\right] \\
& =\frac{(\alpha+\delta)^{2}+5(\delta \alpha+1)^{2}-20 \delta \alpha}{(1-\delta \alpha)^{2}} ;
\end{aligned}
$$

if we let $\alpha=-\delta$ and $\delta \alpha=-1$, then $\mathbf{K}_{a}=\mathbf{K}$, we also obtain $\left\|\mathbf{K}_{a}\right\|_{F}=\sqrt{5}$ which is the same result as we obtained via the one-parameter solutions, Eqs. (9) and (11)
Remark 1 The minimum-norm solution for feedback matrix may be one of the followings:

$$
\left[\begin{array}{cc}
\frac{3}{2} & \pm \frac{1}{2} \\
\pm \frac{1}{2} & \frac{3}{2}
\end{array}\right],\left[\begin{array}{ll}
1 & 0 \\
0 & 2
\end{array}\right],\left[\begin{array}{ll}
2 & 0 \\
0 & 1
\end{array}\right]
$$

Based on the above discussions, for this system to place poles to $\{-2,-3\}$, it is our opinion rendering the following observations,

Observation 1 The minimum-norm solution of feedback gain matrix can be that of (4), and this normal matrix can also achieve robust pole-placement property.

Observation 2 The feedback gain matrix $\mathrm{K}$, put in the linear parametric form of either Eqs (9) or (11), is equipped with one effective free parameter. $\mathbf{K}, \mathbf{K}_{a}$ and $\mathbf{K}_{b}$ simultaneouly attain the minimum-norm of value $\sqrt{5}$

\section{PRELIMINARY DEFINITIONS}

Consider a linear multivariable system $\{\mathbf{A}, \mathbf{B}$, C\}:

$$
\begin{aligned}
& z \mathbf{x}(t)=\mathbf{A x}(t)+\mathbf{B u}(t), \\
& \mathbf{y}(t)=\mathbf{C x}(t),
\end{aligned}
$$

where $z$ represents the operator $d / d t$ in the continuoustime system, or time-shifting [i.e., $z \mathbf{x}(t):=\mathbf{x}(t+1)$ ] in the discrete-time. The state vector $\mathbf{x}(t) \in \mathfrak{R}^{n}$, control vector $\mathbf{u}(t) \in \mathfrak{R}^{n}$, and output vector $\mathbf{y}(t) \in \Re^{n}$, constant matrices $\mathbf{A}, \mathbf{B}, \mathbf{C}$ are of compatible dimensions. It is assumed that $B$ is of full rank $m$ [no redundant inputs in Eq. (12)], and the pair $\{\mathbf{A}, \mathbf{B}\}$ is completely controllable with controllability index $\mu$.

\section{Pole-placement Problem}

The considered problem is the following. For the Eq. (12), find an LSVF law:

$$
\mathbf{u}(t)=-\mathbf{K} \mathbf{x}(t),
$$

such that the eigenvalues of the closed-loop system $\{\mathbf{A}-\mathbf{B K}, \mathbf{B}\}$ is assigned to the pre-determined selfconjugate set $\Lambda:=\left\{\lambda_{i},(i=1 . . n)\right\}$. This is the so called pole-placement problem (the eigenvalue assignment problem).

\section{Parametric Pole-placement Problem}

In our development, we are to find a class of static controllers, in the form of (13), with feedback 
gain matrix $\mathbf{K}$ equipped with minimum number of effective free parameters. This is addressed herein as parametric pole-placement problem.

\section{Robust Pole-placement}

The robust pole-placement design is achieved by finding a feedback gain matrix $\mathbf{K}$ with minimum Frobenius norm to achieve pole-placement purpose.

\section{Minimum-time Deadbeat Control (MTDC) Problem}

If the desired poles in the pole-placement problem are all chosen to be at the origin, for a linear discrete-time system $\{\mathbf{A}, \mathbf{B}, \mathbf{C}\}$, the closed-loop system matrix $\mathbf{A}_{\mathbf{c}}$ := A-BK satisfies

$$
\mathbf{A}_{c}^{v}=0
$$

for some least integer $v$. This strategy has the property of driving all the states to zero in at most $v$ steps, and remain zero afterwards, after an impulse disturbance in the process state. The control strategy is the so called $M T D C$ [17].

\section{Minimum-gain Minimum-time Deadbeat Control (MGMTDC) Problem}

A minimum Frobenius-norm solution is chosen for the feedback gain matrix $K$ in order to achieve MTDC.

Remark 2 All of the above mentioned are basically addressed as the generic pole-placement problem.

\section{THEORY OF HYPOTHETICAL LOOP-DECOUPLING}

The objective of the present paper is to design parametric pole-placement via the hypothetical loop-decoupling approach $[1,18]$. Theoretical stems to be needed for the development of the paper are summarized as followings,

We use compact notations as

$$
\mathbf{B}=\left[\begin{array}{llll}
\mathbf{b}_{1} & \mathbf{b}_{2} & \ldots & \mathbf{b}_{m}
\end{array}\right]:=\underset{j=1 \ldots m}{\operatorname{row}}\left\{\mathbf{b}_{j}\right\},
$$

also,

$$
\mathbf{C}=\left[\begin{array}{c}
\mathbf{c}_{1} \\
\mathbf{c}_{2} \\
\vdots \\
\vdots \\
\mathbf{c}_{q}
\end{array}\right]:={ }_{i=1 \ldots q}^{\operatorname{col}}\left\{\mathbf{c}_{i}\right\}
$$

For the Eq (12), with controllability index $\mu$, the following controllability matrix is of full rank:

$$
\mathbf{M}^{*}=\left[\begin{array}{lllll}
\mathbf{B} & \mathbf{A B} & \ldots & \mathbf{A}^{\mu-1} \mathbf{B}
\end{array}\right] .
$$

By the second scheme of crate search [19] for the matrix $\mathbf{M}^{*}$, one gets a set of integers $\mu_{i},(i=1 \ldots m)$, with

$$
\mu:=\max _{i}\left\{\mu_{i}\right\},
$$

and

$$
\sum_{i=1}^{m} \mu_{i}=n
$$

such that the following matrix, the controllability basis matrix,

$$
\mathbf{M}:=\underset{j=1 . . m}{\operatorname{row}}\left\{\underset{k=0 . . \mu_{j}-1}{\operatorname{row}}\left\{\mathbf{A}^{k} \mathbf{b}_{j}\right\}\right\} \in \mathfrak{R}^{n \times n},
$$

is invertible.

Note that the choice of $\left\{\mu_{i}\right\}$ is not unique as long as $n$ linearly independent vectors, the regular vectors, are chosen to form the nonsingular matrix M. The choice of $\left\{\mu_{i}\right\}$, though not unique, plays the key role in the hypothetical loop-decoupling scheme, however, they have to be constrained by Eqs. (16)(18). The class of parametrized static feedback controllers is also depicted by the set $\left\{\mu_{i}\right\}$ in the paper.

\section{Hypothetical Output}

Hypothetical output was first proposed by Juang [1] as an innovative method in the LSVF design for a linear multivariable system. The hypothetical output for Eq. (12) is defined as

$$
\theta(t)=\underset{i=1 \ldots m}{c o l}\left\{\theta_{i}(t)\right\}=\mathbf{H x}(t) .
$$

Here,

$$
\mathbf{H}:=\underset{i=1 . m}{\operatorname{col}}\left\{\mathbf{h}_{i}\right\} \in \mathfrak{R}^{m \times n} \backslash\{0\},
$$

and $\mathbf{h}_{i}$, plays as the role of observability probe, is a row $n$-vector. The purpose of this choice for $\mathbf{H}$ is to make the system $\{\mathbf{A}, \mathbf{B}, \mathbf{H}\}$ preserve observability from the hypothetical output $\theta(t)$ under any state feedback design via the loop-decoupling framework. The transfer function matrix (TFM) from $\mathbf{u}$ to $\theta$ is all-pole [18], i.e., the TFM contains no finite zeros. The choice of $\mathbf{H}$ is as follows,

Lemma $1[1,18]$ There exists a paticular $\mathbf{H}$ such that 
$\mathbf{h}_{i} \mathbf{A}^{k} \mathbf{b}_{j}=\left\{\begin{array}{cc}1 & \text { if } i=j, k=\mu_{i}-1,(i, j=1 \ldots m) \\ 0 & \text { if } i \neq j \text { while } k \leq \mu_{i}-1 \text { or if } i=j \\ & \text { while } k<\mu_{j}-1 .\end{array}\right.$

proof: Claiming

$$
\mathbf{E}_{\mu}:=\text { block } \operatorname{diag}_{i=1 . . m}\left\{\mathbf{E}_{i}\right\},
$$

where, $\mathbf{E}_{i}$ is the $\mu_{i}$-th unit row vector, so that $\mathbf{H}$ can satisfy

$$
\mathbf{H} \mathbf{M}=\mathbf{E}_{\mu},
$$

therefore,

$$
\begin{aligned}
& \underset{i=1 . . m}{\operatorname{col}}\left\{\mathbf{h}_{i}\right\}\left[\begin{array}{l}
\underset{j=1 \ldots m}{\operatorname{row}}\left\{\left[\begin{array}{lllll}
\mathbf{b}_{j} & \mathbf{A} \mathbf{b}_{j} & \ldots & \mathbf{A}^{\mu_{j}-1} \mathbf{b}_{j}
\end{array}\right]\right.
\end{array}\right] \\
& =\text { block } \operatorname{diag}_{i=1 . . m}\left\{\left[\begin{array}{llllll}
0 & \ldots & 0 & \ldots & 1 & 1
\end{array}\right]\right\} .
\end{aligned}
$$

This proves the lemma. q.e.d.

By this lemma, one has

$$
\mathbf{h}_{i} \mathbf{A}^{k} \mathbf{B}=0, \quad \text { if } k<\mu_{i}-1
$$

and

$$
\mathbf{h}_{i} \mathbf{A}^{\mu_{j}-1} \mathbf{B}=\mathbf{B}_{i}^{*}:=\left[0 \ldots{ }_{i-t h}^{1} \# \ldots \#\right] .
$$

Here \# represents some constant real number, and $\mathbf{B}_{i}^{*}$ a row $m$-vector. Note that Eq. (24) is the set of hypothetical output constraints such that every choice of $\mathbf{h}_{\mathrm{i}}$ has to meet. Therefore, for $i=1 . . m$, each set of subsystems with hypothetical output $\theta_{i}(\mathrm{t})$ and of $\mu_{i}$-th order, is in phase variable form, and is described by

$$
\begin{aligned}
& \theta_{i}(t)=\mathbf{h}_{i} \mathbf{x}(t), \\
& z \theta_{i}(t)=\mathbf{h}_{i} \mathbf{A} \mathbf{x}(t), \\
& \cdots \\
& z^{\mu_{i-1}} \theta_{i}(t)=\mathbf{h}_{i} \mathbf{A}^{\mu_{i}-1} \mathbf{x}(t), \\
& z^{\mu_{i}} \theta_{i}(t)=\mathbf{h}_{i} \mathbf{A}^{\mu_{i}} \mathbf{x}(t)+\mathbf{B}_{i}^{*} \mathbf{u}(t) .
\end{aligned}
$$

One immediately has

$$
\Psi(z) \theta(t)=\Xi \mathbf{x}(t),
$$

where

$$
\Psi(z):=\text { block } \underset{i=1 \ldots m}{\operatorname{diag}}\left\{\operatorname{col}_{k=0 \ldots \mu_{i}-1}\left\{z^{k}\right\}\right\},
$$

and

$$
\left.\Xi:=\operatorname{col}_{i=1 \ldots m k=0 \ldots \mu_{i}-1}^{\operatorname{col}}\left\{\mathbf{h}_{i} \mathbf{A}^{k}\right\}\right\} .
$$

Lemma 2 [18] 1. $\Xi \in \Re^{n \times n}$ is nonsingular. 2. $\mathbf{B}^{*}:=\operatorname{col}_{i=1 . . m}\left\{\mathbf{B}_{i}^{*}\right\} \in \Re^{m \times m}$ is non-
singular.

Proposition 3 The following statements are true:

1. The system $\{\mathbf{A}, \mathbf{B}, \mathbf{H}\}$ is observable.

2. The TFM from $\mathbf{u}$ to $\theta$ is all-pole. proof: The proof follows directly from lemma 2 , and Eqs. (25) and (26). q.e.d.

\section{Generalized Controllable Canonical Form (GCCF)}

We now relate the set of hypothetical subsystems as described by Eq. (25) to GCCF[16,19]. Define

$$
\begin{aligned}
\overline{\mathbf{X}}(t) & =\underset{i=1 . . m}{\operatorname{col}}\left\{{ }_{k=1 \ldots \mu_{i}} \operatorname{col}\left\{\bar{x}_{i k}(t)\right\}\right\}, \\
& =\underset{i=1 . . m}{\operatorname{col}}\left\{{ }_{k=1 \ldots \mu_{i}-1} \operatorname{col}_{k}\left\{z^{k} \theta_{i}(t)\right\}\right\}=\Psi(z) \theta(t),
\end{aligned}
$$

and by Eq. (26) one gets

$$
\overline{\mathbf{x}}(t)=\mathbf{\Xi} \mathbf{x}(t),
$$

which is the state vector transform (change of state variables). By lemma 2, a matrix $L:=\operatorname{col}_{i=1 \ldots m}\left\{l_{i}\right\} \in$ $\mathfrak{R}^{m \times n}$ can be found such that the following linear combination holds:

$$
\underset{i=1}{\operatorname{col}}\left\{\mathbf{h}_{i} \mathbf{A}^{\mu_{i}}\right\}=\boldsymbol{L} \Xi .
$$

Therefore,

$$
\begin{aligned}
& \underset{i=1 \ldots m}{\operatorname{col}}\left\{z^{\mu_{i}} \theta_{i}(t)-\mathbf{B}_{i}^{*} \mathbf{u}(t)\right\}=L \Xi \mathbf{x}(t) \\
& =\underset{i=1 ., m}{\operatorname{col}}\left\{l_{i}\right\} \overline{\mathbf{x}}(t)
\end{aligned}
$$

i.e.,

$$
z^{\mu_{i}} \theta_{i}(t)=l_{i} \overline{\mathbf{x}}(t)+\mathbf{B}_{i}^{*} \mathbf{u}(t) .
$$

Following Eqs. (29) and (32), we have

$$
\begin{aligned}
& z \bar{x}_{i 1}(t)=z \theta_{i}(t)=\bar{x}_{i 2}(t), \\
& z \bar{x}_{i 2}(t)=z^{2} \theta_{i}(t)=\bar{x}_{i 3}(t) \text {, } \\
& \bar{z} \bar{x}_{i \mu_{i}}(t)=z^{\mu_{i} \theta_{i}}(t)=l_{i} \overline{\mathbf{x}}(t)+\mathbf{B}_{i}^{*} \mathbf{u}(t),
\end{aligned}
$$

for $i=1 . . m$. Clearly, Eq. (33) describes the transformed system in GCCF. This is a nice form for doing multi-input pole-placement design, as in the traditional discipline. One immediately obtains the following result.

Proposition 4 The system $\left\{\Xi A \Xi^{-1}, \Xi B, H\right\}$ is in 


\section{GCCF.}

proof: (omitted).

Remark 3 PVBC (phase variable block Canonical form) has been suggested by Fahmy and O'Reilly [20] in doing parametrization of MTDC. It is seen that GCCF is actually one kind of this concept.

\section{General Pole-placement Design}

We now present the pole-placement design using state-feedback via hypothetical loop-decoupling approach. Let

$$
\pi_{i}(z):=z^{\mu_{i}}+\sum_{k=0}^{\mu_{i}-1} a_{k} z^{k},(i=1 . . m)
$$

be the desired characteristic polynomial for the $i$-th control loop whose hypothetical output is $\theta_{i}(t)$, flowing Eq. (25) one has

$$
\underset{i=1 . . m}{\operatorname{col}}\left\{\pi_{i}(z) \theta_{i}(t)\right\}=\mathbf{A}^{*} \mathbf{x}(t)+\mathbf{B}^{*} \mathbf{u}(t),
$$

here

$$
\mathbf{A}^{*}=\underset{i=1 \ldots m}{\operatorname{col}}\left\{\mathbf{h}_{i} \pi_{i}(\mathbf{A})\right\},
$$

and,

$$
\mathbf{B}^{*}=\underset{i=1 \ldots m}{\operatorname{col}}\left\{\mathbf{B}_{i}^{*}\right\} .
$$

Setting $\mathbf{A}^{*} \mathbf{x}(t)+\mathbf{B} * \mathbf{u}(t):=\mathbf{v}(t), \mathbf{v}$ a new control, and get

$$
\begin{aligned}
\mathbf{u}(t) & =\left[\mathbf{B}^{*}\right]^{-1} \mathbf{v}(t)-\left[\mathbf{B}^{*}\right]^{-1} \mathbf{A}^{*} \mathbf{x}(t) \\
& :=\mathbf{F v}(t)-\mathbf{K x}(t)
\end{aligned}
$$

as the LSVF law achieving the determined poleplacement task. Following Eqs (35) and (37), the hypothetical loop-decoupling is depicted by,

$$
\pi_{i}(z) \theta_{i}(t)=v_{i}(t), \quad(i=1 . . m)
$$

From above discussion, one immediately has the following theorem:

Theorem 5 [18] (Generalized Ackermann's Formula)

A linear time-invariant controllable Eq. (12) can always be loop-decoupled, with pole-placement as specified by Eq. (34), by LSVF law Eq. (37) with the feedback gain matrix:

$$
\mathbf{K}^{*}=\left[\mathbf{B}^{*}\right]^{-1} \underset{i=1 \ldots m}{\operatorname{col}}\left\{\mathbf{h}_{i} \mathbf{A}^{\mu_{i}}\right\} .
$$

here, $B^{*}$ and $A^{*}$ are defined as by Eq. (36). proof: (omitted).

Remark 4 To obtain stable numerical results, the computation of $\mathbf{h}_{i} \pi_{i}(\mathbf{A})$ is suggested to proceed as follows

$$
\mathbf{h}_{i} \pi_{i}(\mathbf{A})=\left\{\left[\left(\left(\mathbf{h}_{i}\right) \mathbf{A}+a_{\mu_{i}-1} \mathbf{h}_{i}\right) \mathbf{A}+\ldots\right] \mathbf{A}+a_{1} \mathbf{h}_{i}\right\} \mathbf{A}+a_{0} \mathbf{h}_{i}
$$

Remark 5 We have $\mathrm{n}$ closed-loop poles arranged into $m$ groups, each defined by $\pi_{i}(z)=0$ for $\mu_{i}$ poles. This also offers flexibility in choosing the gain matrix $\mathbf{K}$.

\section{MTDC Design}

MTDC (minimum-time deadbeat control) design is achieved by setting all closed-loop poles to the origin $(z=0)$, by Eq. (25) let

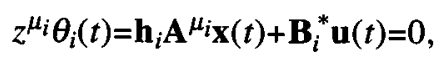

then $z^{\mu_{i}} \theta_{i}(t)=0$ for all $i=1 . . m$. Hence $\theta_{i}(t)=0$ for all $t \geq \mu$ implies that $\mathbf{x}(t)=\mathbf{0}$ and also $\mathbf{y}(t)=\mathbf{0}$ for all $t \geq \mu$ Since $\mu$ is the least integer such that the controllability matrix $\mathbf{M}$ can be of full rank $n$, so MTDC is obtained.

The following theorem is ready to state:

Theorem 6 [17] (MTDC Control Design)

For the linear controllable discrete-time Eq. (12), the MTDC feedback control law is $\mathbf{u}(t)=-\mathbf{K}^{*} \mathbf{x}(t)$, where

$$
\mathbf{K}^{*}=\left[\mathbf{B}^{*}\right]^{-1} \underset{i=1 . . m}{\operatorname{col}}\left\{\mathbf{h}_{i} \mathbf{A}^{\mu_{i}}\right\} .
$$

proof: This follows Eq. (41) directly. q.e.d.

\section{PARAMETRIZATION}

\section{Linear Parametrization}

Lemma 7 Let

$$
\overline{\mathbf{h}}_{i}=\mathbf{h}_{i}+\sum_{\substack{j \\ \mu_{j}>\mu_{i}}}^{\mu_{j}-\mu_{i}-1} \sum_{k=0} \alpha_{i k j} \mathbf{h}_{j} \mathbf{A}^{k},
$$

here $\alpha_{i k j}$ 's are free parameters, $\mathbf{h}_{i}{ }^{\prime}$ s are those probes as determined as in lemma 1 , then $\bar{h}_{i}^{\prime}$ s can also satisfy the hypothetical output constraints Eq. (24), i.e.,

$$
\overline{\mathbf{h}}_{i} \mathbf{A}^{k} \mathbf{B}=\left\{\begin{array}{ll}
\mathbf{0} & \text { if } k<\mu_{i}-1 \\
\mathbf{B}_{i}^{*} & \text { if } k=\mu_{i}-1
\end{array},\right.
$$

where $\mathbf{0}$ denotes a zero row vector. 
proof: (see appendix A)

By these linearly parametrized probes $\overline{\mathbf{h}}_{i}^{\prime}$ s, then $\mathbf{A}^{*}$ in theorem 5, [Eq. (39)], is reiterated as

$$
\mathbf{A}^{*}=\underset{i=1 . . m}{\operatorname{col}}\left\{\overline{\mathbf{h}}_{i} \pi_{i}(\mathbf{A})\right\}
$$

and the feedback gain matrix is

$$
\overline{\mathbf{K}}=\left[\mathbf{B}^{*}\right]^{-1} \mathbf{A}^{*},
$$

here, $\overline{\mathbf{K}}$ is equipped with parameters coupled in linear function form.

Corollary 8 The number of free parameters for linear parametrization is

$$
N_{p}=\frac{1}{2} \sum_{i} \sum_{j}\left|\mu_{i}-\mu_{j}\right|
$$

proof: By Eq. (43) we have

$$
N_{p}=\frac{1}{2} \sum_{i} \sum_{\substack{j \\ \mu_{j} \geq \mu_{i}}}\left(\mu_{i}-\mu_{j}\right),
$$

therefore, Eq. (45) follows directly. q.e.d.

Remark 6 Let $\{\mu(i),(i=1 . . m)\}=\left\{\mu_{i},(i=1 . . m)\right\}$ such that $\mu(i) \geq \mu(i+1)$, then

$$
N_{p}=m m-\sum_{k=1}^{m}(2 k-1) \mu(k) .
$$

This is also the result given by Amim et. al [24], Elabdalla et. al. [13], and Schelegel [10].

Let

$$
\mathbf{A}^{*}=\underset{i=1 . . m}{\operatorname{col}}\left\{\overline{\mathbf{h}}_{i} \pi_{i}(\mathbf{A})\right\},
$$

and consider

$$
\overline{\mathbf{H}} \mathbf{M}=\overline{\mathbf{E}}:=\left[\overline{\mathbf{E}}_{i j}\right],
$$

where

$$
\overline{\mathbf{E}}_{i j}=\underset{k=0 . . \mu_{j}-1}{\operatorname{row}}\left\{\bar{E}_{i k j}\right\},
$$

and

$$
\bar{E}_{i k j}=\overline{\mathbf{h}}_{i} \mathbf{A}^{k} \mathbf{b}_{j}
$$

For some $i, j$ such that $\mu_{j}>\mu_{i}, \overline{\mathbf{h}}_{i} \mathbf{A}^{k} \mathbf{b}_{j}$ has no constraint while $\mu_{i} \leq k \leq \mu_{j}-1$, and can be any free number (the free parameter).

Based on the above discussion, by modifying lemma 1, the generalized probes can be defined as follows:

Proposition 9 The generalized probes $\overline{\mathbf{h}}_{i}^{\prime}$ s can be chosen such that,

$$
\overline{\mathbf{h}}_{i} \mathbf{A}^{k} \mathbf{b}_{j}= \begin{cases}1 & \text { if } i=j, k=\mu_{i}-1,(i, j=1 . . m) \\ 0 & \text { if } i=j \text { while } k<\mu_{i}-1, \text { or } \\ & \text { if } i \neq j, \text { while } k<\min \left\{\mu_{i}, \mu_{j}\right\}-1 \\ \beta_{i k j} & \text { (free) if } i \neq j \text { and } \mu_{j}>\mu_{i} \\ & \text { while } \mu_{i} \leq k \leq \mu_{j}-1\end{cases}
$$

proof: (omitted).

Remark 7 Linear parametrization for gain matrix is possible when the matrix $\mathbf{B}^{*}$ contains no free parameters in it. Note that this is sufficient, but not necessary in general.

\section{Nonlinear Parametrization}

We have shown that, via hypothetical loopdecoupling approach, linear parametrization in gain matrix is possible if the generalized probes $\overline{\mathbf{h}}_{i}^{\prime}$ s are chosen based on proposition 9[Eq. (50)], and the matrix $\mathbf{B}_{i}^{*}$ is constant and invertible. This is always attainable if we choose $N_{p}$ free parameters [Eq. (45)] in $\overline{\mathbf{E}}$ [eqn.(49)] or by embedding the parameters in $\overline{\mathbf{h}}_{i}^{\prime}$ s as defined by lemma 7 .

It is also possible to embed more free parameters in $\overline{\mathbf{h}}_{i}$ along with other $\mathbf{h}_{j}^{\prime} s$ in which $\mu_{j}=\mu_{i}$, see the motivation example section of this paper. This will in general result in parameters nonlinearly coupled in the gain matrix. The fact that free parameters appear in both $\mathbf{B}^{*}$ and $\mathbf{A}^{*}$ may result in that the feedback gain matrix $\overline{\mathbf{K}}$ nonlinearly parametrized. This is what splendid as disclosed in the paper and is something new to the control society.

Under our investigation, it is believed that, if there are $p_{l}$ hypothetical control loops equipped with the same controllability index number, say $\mu_{l}$, then there can be

$$
N_{0}(l):=\frac{p_{l}\left(p_{l}-1\right)}{2},
$$

more free parameters for use in the difinition of $\overline{\mathbf{h}}_{i}^{\prime}$ s or $\overline{\mathbf{E}}$. Putting together of them, let

$$
N_{0}:=\sum_{l} N_{0}(l)
$$

we have the following result:

Proposition 10 The total number of free parameters in doing nonlinear parametrization for the gain matrix is

$$
N_{n}=N_{p}+N_{0}
$$

proof: (omitted). 
Remark 8 It should be aware that if one also assigns the same $\mu_{l}$ poles for these $p_{l}$ control loops (same loop characteristics), then those extra parameters become not effective. In this very special case, parametrization turns out to be linear. We will clarify this fact by later illustrative examples.

To enforce the above investigation for special cases, consider a system as follows:

$$
z \mathbf{X}(t)=\left[\begin{array}{ccc}
-1 & 0 & 0 \\
0 & -1 & 0 \\
0 & 0 & -1
\end{array}\right] \mathbf{x}(t)+\left[\begin{array}{lll}
1 & 0 & 0 \\
0 & 1 & 0 \\
0 & 0 & 1
\end{array}\right] \mathbf{u}(t)
$$

we have $\mu_{1}=\mu_{2}=\mu_{3}=1$, and $N_{p}=0 ; p_{1}=3, N_{0}=3$, therefore $N_{n}=3$. So we are expecting three free parameters to be equipped as follows:

$$
\overline{\mathbf{E}}=\left[\begin{array}{ccc}
1 & \alpha_{1} & \alpha_{2} \\
0 & 1 & \alpha_{3} \\
0 & 0 & 1
\end{array}\right]=\overline{\mathbf{H}}=\mathbf{B}^{*}
$$

The feedback gain matrix used to place poles to $\{-1,-$ $2,-3$ \} is found to be

$$
\mathbf{K}=\left[\begin{array}{ccc}
0 & -\alpha_{1} & \alpha_{1} \alpha_{3}-2 \alpha_{2} \\
0 & 1 & -\alpha_{3} \\
0 & 0 & 2
\end{array}\right]
$$

by Eq. (39), in which parameters seem to be nonlinearly coupled. However, by setting $\alpha=-\alpha_{1}, \beta=-\alpha_{2}, \delta=\alpha_{1} \alpha_{3}$ $-2 \alpha_{2}$ one has

$$
\mathbf{K}=\left[\begin{array}{lll}
0 & \alpha & \delta \\
0 & 1 & \beta \\
0 & 0 & 2
\end{array}\right]
$$

which is also linearly parametrized in terms of the free parameters: $\alpha, \beta, \delta$.

For the case of MTDC design (discrete-time), the gain matrix becomes

by Eq. (42), and all parameters becomes not effictive. Therefore $N_{n}=N_{p}=0$ in this MTDC case. This verifies the above conjecture (remark 8).

\section{ILLUSTRATIVE EXAMPLES}

The results of previous section will be illustrated by following examples.

\section{Parametric MTDC Design}

Example 1 For the sake of comparison, consider the discrete-time system used in Amin et. al.[14] and Elabdalla et. al. [13], for the design of MTDC con- troller, where

$$
A=\left[\begin{array}{cccc}
1 & 2 & 0 & 0 \\
0 & -2 & 0 & 1 \\
1 & 1 & 0 & 1 \\
-1 & -1 & 0 & 0
\end{array}\right], B=\left[\begin{array}{lll}
1 & 0 & 0 \\
0 & 1 & 0 \\
0 & 0 & 1 \\
0 & 0 & 0
\end{array}\right],
$$

hence $n=4, m=3$, and one can find $\mu=2$. We consider two classes as follows:

class (a): $\mu_{1}=2, \mu_{2}=1, \mu_{3}=1$, so that

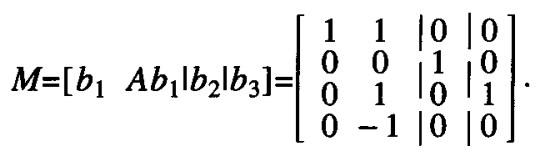

Because $\mu_{1}-\mu_{2}=1, \mu_{1}-\mu_{3}=1$, we have $N_{p}=2$ free parameters to use. Following Eq. (50),

$$
\bar{E}:=\left[\begin{array}{cc|c|c}
0 & 1 & 0 & 0 \\
\hline 0 & \alpha & 1 & 0 \\
\hline 0 & \beta & 0 & 1
\end{array}\right],(\alpha, \beta \in \Re)
$$

so that,

$$
\begin{aligned}
& \bar{H}=\bar{E} M^{-1}=\left[\begin{array}{l}
\frac{0}{0}-\frac{0}{1}-\frac{0}{0}-\frac{-1}{-\alpha}+; \\
\frac{0}{0}-\beta
\end{array}\right] \text { and } \\
& B^{*}=\left[\begin{array}{lll}
1 & 1 & 0 \\
0 & 1 & 0 \\
0 & 0 & 1
\end{array}\right] \text {. }
\end{aligned}
$$

Following Eq. (42), the class of MTDC law $u(t)$ $=-\bar{K}_{a} x(t)$ is represented by

$$
\bar{K}_{a}=\left[\begin{array}{cccc}
1-\alpha & 2-\alpha & 0 & 0 \\
\alpha & -2+\alpha & 0 & 1 \\
\beta & \beta & 0 & 1
\end{array}\right] .
$$

If we set $\alpha=-p_{1}, \beta=1-p_{2},\left(p_{1}, p_{2}\right.$ are free $)$, then $\bar{K}_{a}$ becomes

$$
\bar{K}_{a}=\left[\begin{array}{cccc}
1+p_{1} & 2+p_{1} & 0 & 0 \\
p_{1} & -2+p_{1} & 0 & 1 \\
1-p_{2} & 1-p_{2} & 0 & 1
\end{array}\right]
$$

which is just the result as $\bar{K}_{a}$, given by [14]. The closed-loop system matrix is

$$
A_{c}=A-B \bar{K}_{a}=\left[\begin{array}{cccc}
\alpha & \alpha & 0 & 0 \\
-\alpha & -\alpha & 0 & 0 \\
1-\beta & 1-\beta & 0 & 0 \\
-1 & -1 & 0 & 0
\end{array}\right] .
$$

It is seen that $A_{c}^{\mu}=A_{c}^{2}=0$. Let an arbitrary initial state be $\mathbf{x}(0)=\left[\begin{array}{llll}a & b & c & d\end{array}\right]^{T}$, then $\mathbf{x}(1)=(a+b)\left[\begin{array}{lll}\alpha-\alpha & 1-\end{array}\right.$ $\beta-1]^{T}$, and $\mathbf{x}(2)=\mathbf{x}(\mu)=0$. Therefore, $\mathbf{x}(k)=0$ for all $k \geq \mu=2$, This verifies that a 2-parameter solution can accomplish MTDC task. 
Example 2 (minimum effective parameters)

Consider again the system of example 1, we here use over-parametrization by putting one more free parameter $\delta$ in $\bar{E}$, [class (a)], as follows:

$$
\bar{E}:=\left[\begin{array}{llll}
0 & 1 & 0 & 0 \\
0 & \alpha & 1 & \delta \\
0 & \beta & 0 & 1
\end{array}\right],(\alpha, \beta, \delta \in \Re)
$$

so that

$$
\begin{aligned}
\bar{H}=\bar{E} M^{-1} & =\left[\begin{array}{cccc}
0 & 0 & 0 & -1 \\
0 & 1 & \delta & -\alpha+\delta \\
0 & 0 & 1 & 1-\beta
\end{array}\right] \\
& :=\left[\begin{array}{c}
\bar{h}_{1} \\
\bar{h}_{2} \\
\bar{h}_{3}
\end{array}\right] .
\end{aligned}
$$

One may show that the probe $\bar{h}_{i}^{\prime}$ s still satisfy the hypothetical output constraints as given by Eq. (44). Following Eq. (42), the LSVF law for MTDC is represented by

$$
\bar{K}=\left[\begin{array}{cccc}
1-\alpha+\delta \beta & 2-\alpha+\delta \beta & 0 & 0 \\
\alpha-\delta \beta & -2+\alpha-\delta \beta & 0 & 1 \\
\beta & \beta & 0 & 1
\end{array}\right],
$$

setting $\alpha-\delta \beta=-p_{1}$ and $\beta=1-p_{2}$, then

$$
\bar{K}=\left[\begin{array}{cccc}
1+p_{1} & 2+p_{1} & 0 & 0 \\
-p_{1} & -2-p_{1} & 0 & 1 \\
1-p_{2} & 1-p_{2} & 0 & 1
\end{array}\right]
$$

which is a 2-parameter solution, and is just the result as what we have, also in [20]. It is then clear that the minimum number of effective free parameters is 2 , but not more than 2 . This clarifies the conjecture made in remark 8 of the paper.

\section{Parametric Pole-placement Design}

Example 3 Consider a linear continuous-time system $\{\mathbf{A}, \mathbf{B}, \mathbf{C}\}$ with system matrices $\mathbf{A}, \mathbf{B}$ given as in example 1 (also in [14] and [13]). We are to find a feedback gain matrix $\mathbf{K}$, equipped with several free parameters, such that the LSVF law $u(t)=v(t)-K x(t)$ can place closed-loop poles to the set $\Lambda:=\{-1,-2$, $-3,-4\}$. There are two control classes for this system: (a) $\mu_{1}=2, \mu_{2}=\mu_{3}=1$; (b) $\mu_{2}=2, \mu_{1}=\mu_{3}=1$. (Note that $\mu_{1}=\mu_{2}=1, \mu_{3}=2$ is not possible, because the controllability matrix $M$, as defined by Eq. (18), is singular for this class).

We consider class (a) with $\pi_{1}(z)=(z+1)(z+2)$, $\pi_{2}(z)=(z+3), \pi_{3}(z)=(z+4)$ as the loop-assignment.
(Note: There are 12 choices of assigning these four distinct poles into three hypothetical control loops.) Because $\mu_{1}-\mu_{2}=1, \mu_{1}-\mu_{3}=1$, and $\mu_{3}=\mu_{2}$, we suggest 3 free parameters in the following two cases: (case 1): Let

$$
\begin{aligned}
\bar{K}_{a} & =\left[\begin{array}{llll}
0 & 1 & 0 & 0 \\
0 & \alpha & 1 & \delta \\
0 & \beta & 0 & 1
\end{array}\right], \text { hence } \\
\bar{H}_{a} & =\bar{E}_{a} M^{-1}=\left[\begin{array}{lllc}
0 & 0 & 0 & -1 \\
0 & 1 & \delta & -\alpha+\delta \\
0 & 0 & 1 & 1-\beta
\end{array}\right] .
\end{aligned}
$$

Observing the phase variables of hypothetical outputs respectively as follows:

$$
\begin{aligned}
& \theta_{1}(t)=\left[\begin{array}{llll}
0 & 0 & 0 & -1
\end{array}\right] x(t), \\
& z \theta_{1}(t)=\left[\begin{array}{llll}
1 & 1 & 0 & 0
\end{array}\right] x(t), \\
& z_{2} \theta_{1}(t)=\left[\begin{array}{lllll}
1 & 0 & 0 & 1
\end{array}\right] x(t)+\left[\begin{array}{lll}
1 & 1 & 0
\end{array}\right] u(t), \\
& \theta_{2}(t)=\left[\begin{array}{llll}
0 & 1 & \delta & -\alpha+\delta
\end{array}\right] x(t), \\
& z \theta_{1}(t)=\left[\begin{array}{lllll}
\alpha & \alpha-2 & 0 & 1+\delta
\end{array}\right] x(t)+\left[\begin{array}{lll}
0 & 1 & \delta
\end{array}\right] u(t), \\
& \theta_{3}(t)=\left[\begin{array}{lllll}
0 & 0 & 1 & 1-\beta
\end{array}\right] x(t), \\
& z \theta_{3}(\mathrm{t})=\left[\begin{array}{lllll}
\mathrm{b} & \mathrm{b} & 0 & 1
\end{array}\right] x(t)+\left[\begin{array}{llll}
0 & 0 & 1
\end{array}\right] u(t) .
\end{aligned}
$$

By the required loop pole-assignment, set

$$
\begin{aligned}
& \left(z_{2}+3 z+2\right) \theta_{1}(t)=\left[\begin{array}{llll}
4 & 0 & 0 & 2
\end{array}\right] x(t)+\left[\begin{array}{lll}
1 & 1 & 0
\end{array}\right] u(t), \\
& (z+3) \theta_{2}(t)=\left[\begin{array}{llll}
\alpha & \alpha+1 & 3 \delta & 1+4 \delta-3 \alpha) x(t)
\end{array}\right. \\
& +\left[\begin{array}{lll}
0 & 1 & \delta
\end{array}\right] u(t) \text {, } \\
& (z+4) \theta_{3}(t)=\left[\begin{array}{llll}
\beta & \beta & 4 & 5-4 \beta
\end{array}\right] x(t)+\left[\begin{array}{lll}
0 & 0 & 1
\end{array}\right] u(t) \text {. }
\end{aligned}
$$

Following Eq. (39a), the feedback gain matrix, equipped with 3 free parameters, is

$$
\begin{aligned}
& K_{a}=\left[\begin{array}{lll}
1 & 1 & 0 \\
0 & 1 & \delta \\
0 & 0 & 1
\end{array}\right]^{-1}\left[\begin{array}{crlr}
4 & 0 & 0 & 2 \\
\alpha & \alpha+1 & 3 \delta & 1+4 \delta-3 \alpha \\
\beta & \beta & 4 & 5-4 \beta
\end{array}\right], \\
& =\left[\begin{array}{cccc}
4-\bar{\alpha} & -(1+\bar{\alpha}) & \delta & 1+3 \bar{\alpha}+\delta(1-\beta) \\
\alpha & 1+\bar{\alpha} & \delta & 1-3 \bar{\alpha}-\delta(1-\beta) \\
\beta & \beta & 4 & 5-4 \beta
\end{array}\right]
\end{aligned}
$$

Here, $\bar{\alpha}:=\alpha-\delta \beta, \delta, \beta$ are all free, and we have parameters coupling in nonlinear function forms.

(case 2): Otherwise let

$$
\begin{aligned}
& \bar{E}_{b}=\left[\begin{array}{ll|ll}
0 & 1 & 0 & 0 \\
\hline 0 & \alpha & 1 & 0 \\
\hline 0 & \beta & \mid \varepsilon & 11
\end{array}\right], \text { hence }
\end{aligned}
$$

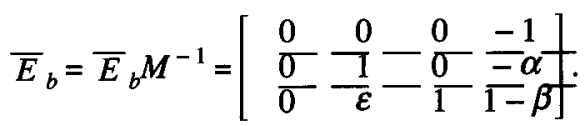

By manipulating hypothetical loop-decoupling 
procedures as in case (a), one gets the feedback gain matrix (also equipped with three free parameters) as follows:

$$
K_{b}=\left[\begin{array}{cccc}
4-\alpha & -(1+\alpha) & 0 & 1+3 \alpha \\
\alpha & 1+\alpha & 0 & 1-3 \alpha \\
\bar{\beta} & \bar{\beta}+\varepsilon & 4 & 5-4 \bar{\beta}-\alpha \varepsilon
\end{array}\right] .
$$

Here, $\bar{\beta}:=\beta-\alpha \varepsilon, \alpha$, and $\mathrm{e}$ are all free. It is seen that both $K_{a}$ and $K_{b}$ are equipped with three free parameters coupling in nonlinear form. It is also interesting to note that , by setting $\delta=0$ for $K_{a}$ or $\varepsilon=0$ for $K_{b}$, then the feedback gain matrix becomes

$$
K=\left[\begin{array}{cccc}
4-\alpha & -(1+\alpha) & 0 & 1+3 \alpha \\
\alpha & 1+\alpha & 0 & 1-3 \alpha \\
\beta & \beta & 4 & 5-4 \beta
\end{array}\right],
$$

which is a two-parameter solution, and most importantly, in linear parametric form. This result may call more valuable applications in optimal control.

Remark 9 We have $K_{a}$ and $K_{b}$, equipped with 3 free parameters coupling in nonlinear form. However, it is no bad using $\mathrm{K}$, equipped with only two effective free parameters in linear form.

Example 4 (minimum number of effective parameters)

Consider again the system of example 2, we are to place all closed-loop poles to -1 . Only the class that $\mu_{1}=2, \mu_{2}=\mu_{3}=1$ is considered here. we use overparametrization by putting four parameters into $\bar{E}$, i.e.,

$$
\bar{E}:=\left[\begin{array}{llll}
0 & 1 & 0 & 0 \\
0 & \alpha & 1 & \delta \\
0 & \beta & \varepsilon & 1
\end{array}\right],(\alpha, \beta, \delta, \varepsilon \in \Re) .
$$

Therefore the probe is

$$
\bar{H}=\bar{E} M^{-1}=\left[\begin{array}{lllc}
0 & 0 & 0 & -1 \\
0 & 1 & \delta & -\alpha+\delta \\
0 & \varepsilon & 1 & 1-\beta
\end{array}\right]:=\left[\begin{array}{c}
\bar{h}_{1} \\
\bar{h}_{2} \\
\bar{h}_{3}
\end{array}\right]
$$

Based on hypothetical loop-decoupling approach, the phase variables of the hypothetical outputs are observed respectively as follows:

$$
\begin{aligned}
& \theta_{1}(t)=\left[\begin{array}{llll}
0 & 0 & 0 & -1
\end{array}\right] x(t), \\
& z \theta_{1}(t)=\left[\begin{array}{llll}
1 & 1 & 0 & 0
\end{array}\right] x(t), \\
& z^{2} \theta_{1}(t)=\left[\begin{array}{llll}
1 & 0 & 0 & 1
\end{array}\right] x(t)+\left[\begin{array}{lll}
1 & 1 & 0
\end{array}\right] u(t), \\
& \theta_{2}(t)=\left[\begin{array}{llll}
0 & 1 & \mathrm{~d} & -\alpha+\delta
\end{array}\right] x(t), \\
& z \theta_{2}(t)=\left[\begin{array}{lllll}
\alpha & \alpha-2 & 0 & 1+\delta
\end{array}\right] x(t)+\left[\begin{array}{lll}
0 & 1 & \delta
\end{array}\right] u(t), \\
& \theta_{3}(t)=\left[\begin{array}{lllll}
0 & \varepsilon & 1 & 1-\beta
\end{array}\right] x(t), \\
& z \theta_{3}(t)=\left[\begin{array}{lllll}
\beta & \beta-2 \varepsilon & 0 & 1+\varepsilon
\end{array}\right] x(t)+\left[\begin{array}{llll}
0 & \varepsilon & 1
\end{array}\right] u(t) .
\end{aligned}
$$

Setting, $\pi_{1}(z)=\pi_{2}+2 z+1, \pi_{2}(z)=\pi_{3}(z)=z+1$, following Eq. (39a), the feedback gain matrix becomes

$$
\begin{aligned}
\bar{K} & =\left[\begin{array}{lll}
1 & 1 & 0 \\
0 & 1 & \delta \\
0 & \varepsilon & 1
\end{array}\right]^{-1}\left[\begin{array}{cccc}
3 & 2 & 0 & 0 \\
\alpha & \alpha-1 & \delta & 1-\alpha+2 \delta \\
\beta & \beta-\varepsilon & 1 & 2-\beta+\varepsilon
\end{array}\right], \\
& =\left[\begin{array}{cccc}
3-\bar{\alpha} & 3-\bar{\alpha} & 0 & \bar{\alpha}-1 \\
\bar{\alpha} & \bar{\alpha}-1 & 0 & -\bar{\alpha}+1 \\
\bar{\beta} & \bar{\beta} & 1 & 2-\bar{\beta}
\end{array}\right] .
\end{aligned}
$$

where, $\bar{\alpha}:=\alpha-\delta \beta$ and $\bar{\beta}:=\beta-\alpha \varepsilon$ are also free. It is seen that the number of effective free parameters is 2 , but not more than 2 , and $\bar{K}$ is a linear parametric form for this example.

Remark 10 We have placed all poles to -1 for this example, and it is shown that the minimum number of effective parameters is $\left(\mu_{1}-\mu_{2}\right)+\left(\mu_{1}-\mu_{3}\right)=2$. This fact has also been shown in example 2, where MTDC design (place all poles to $\mathrm{z}=0$ ) for a discrete-time system (with same system matrices) was given.

\section{CONCLUDING REMARKS}

We will make a concluding remark for the significant work of this paper.

1. An innovative approach based on hypothetical loop-decoupling theory $[1,18]$ for parametrizing a class of state-feedback pole-placement controllers through the minimum number of effective free parameters is presented. The class of control design is depicted by the choice of the set of controllability indices $\left\{\mu_{i}\right\}$ under the framework of the theory. 2.

2. The contribution of the paper is the explicit linear or nonlinear parametric form for the gain matrix $\overline{\mathbf{K}}$ representing the class of controllers for a generic pole-placement design.

3. The hypothetical output probes $\bar{h}_{i}^{\prime}$ s, equipped with effective free parametes, play the key role in our work. When $N_{p}$ parameters [Eq. (45)] are introduced in $\overline{\mathbf{E}}$ (or $\overline{\mathbf{H}}$ ), then $\mathbf{B}^{*}$ is a constant invertible matrix, and $\overline{\mathbf{K}}$ displays the linear parametrization. This was illustrated by example 3. If extra parameters are embedded in $\bar{h}_{i}^{\prime}$ s along with other $\mathbf{h}_{j}$ 's in which $\mu_{j}=\mu_{i}$, then parameters appear in both $\mathbf{B}^{*}$ and $\mathbf{A}^{*}$, this results in that the gain matrix $\overline{\mathbf{K}}$ become nonlinearly parametrized (parameters coupled in nonlinear functions, in the entries of $\overline{\mathbf{K}}$ ). This is what splendid as disclosed in the paper and is new to the control society.

4. Over-parametrization for the pole-placement of equal characteristic polynomial in control loops 
may result in that the extra parameters be not effective, this was illustrated by example 4 , also in examples 1 and 2 where MTDC design was given.

5. Feedback gain matrix parametrization is believed to be helpful in the optimal control, e.g., robust pole-placement design, where parameters may further be tuned for other purpose. This achieves mixed pole-placement and optimal performance design works.

\section{ACKNOWLEDGEMENT}

The author is grateful to the National Science Counsil, R.O.C., for partial financial support under contract no. NSC-86-2213-E-019-004.

\section{REFERENCES}

1. Juang, J.Y., "Introduction to Loop-Decoupling for the Design of Linear Multivariable Systems," Proc. 1988 National Automatic Control Conference, Hsin-chu, ROC, pp. 1-8 (1988).

2. Falb, P.L. and Wolovich, W.A., "Decoupling in the Design and Synthesis of Multivariable Control Systems," IEEE Trans. Automat. Contr., Vol. AC-12, pp. 651-659 (1967).

3. Kalman, R.E., "On the General Theory of Control Systems," Proc. 1st IFAC Congr., London: Butterworth, Vol. 1, pp. 481-492 (1961).

4. Roppenecker G., "On Parametric State Feedback Design," Int. J. Contr., Vol. 43, No. 3, pp. 793-804 (1986).

5. Kautsky, J., Nichols, N.K. and Van Dooren, P. "Robust Pole Assignment in Linear State Feedback," Int. J. Control, Vol. 41, pp.1129-1155 (1985).

6. Dickman, A., "On the Robustness of Multivariable Linear Feedback System in State-space Representation," IEEE Trans. Automat. Contr., Vol. AC-32, No. 5, pp. 407-410 (1987).

7. Fahmy,M.M. and O'Reilly, J., "Comment on a Design of Deadbeat Controllers," IEEE Trans. Automat. Contr., Vol. AC-28, No. 1, pp. 125-127 (1983).

8. O'Reilly, J. and Fahmy, M.M., "The Minimum Number of Degrees of Freedom in State Feedback Design," Int. Journal Contr., Vol.41, No. 3, pp.749-768 (1985).

9. Sebakhy, O.A. and Abdel-Moneim, T.M., "Design of Optimal Dead-Beat Controllers," IEEE Trans. Automat. Contr., Vol. 25, No. 3, pp. 604-606 (1980).

10. Schlegel, M., "Parameterization of the Class of Deadbeat Controllers," IEEE Trans. Automat. Contr., Vol. AC-27, pp. 727-729 (1982).

11. Kucera V., "The Structure Property of Time-Optimal Discrete-Time Linear Control,"IEEE Trans. Automat.
Contr., Vol. AC-16, pp. 375-377 (1971).

12. Amin, M.H., "Construction and Parametrization of a Class of Minimum-Time Deadbeat Controllers,"Systems and Control Letters, Vol. 13, pp. 353-361 (1989).

13. Elabdalla, A.M. and M. H. Amin, "Minimum-Gain minimum-Time Deadbeat Controllers," Systems \& Control Letters, Vol. 11, pp. 213-219 (1988).

14. Amin, M.H. and Elabdalla, A.M.,"Parametrization of a Class of Deadbeat Controllers Via the Theory of Decoupling," IEEE Trans. Automat. Contr., Vol. AC-33, No. 12, pp. 1185-1188 (1988).

15. Amin, M. H., "Parametrization of Minimum-Time Output Deadbeat Controllers for All Classes of Invertible Systems," Int. J. Contr., Vol. AC-52, No. 3, pp. 641-653 (1990).

16. Chen, C.T.,Linear System Theory and Design,H.R.W., N.Y. (1984).

17. Juang, J.Y. and Yu, K.W., "Design of Deadbeat Control via Hypothetical Loop-decoupling," Journal of Art and Science, Vol. 2, National Taiwan Ocean University, pp. 1-15 (1993).

18. Juang, J.Y., "Multivariable Generalization of Ackermann's Formula,"Journal of the Chinese Institute of Engineers (JCIE), Vol. 15 No. 5, pp. 593-604 (1992).

19. Kailath, T., Linear systems, Prentice-Hall (1980).

20. Fahmy, M.M. and O'Reilly, J., "Use of the Design Freedom of Time-Optimal Control," Systems Control Letters, Vol. 3, pp. 23-30 (1983).

\section{APPENDIX}

A. We have the probes hi's defined by lemma 1 and Eq. (23), and the constraint Eq. (24) are satisfied. Let $\mu_{j}>\mu_{i}$ for some $j \neq i$, and let $N(j, i)=\mu_{j}-\mu_{i}$. Consider $k \leq N(j, i)-1$ for

$$
\overline{\mathbf{h}}_{i}=\mathbf{h}_{i}+\alpha_{i k j} \mathbf{h}_{j} \mathbf{A}^{k},
$$

then

$$
\begin{aligned}
\overline{\mathbf{h}}_{i} \mathbf{A}^{\mu_{i}-2} \mathbf{B} & =\mathbf{h}_{i} \mathbf{A}^{\mu_{i}-2} \mathbf{B}+\alpha_{i k j} \mathbf{h}_{j} \mathbf{A}^{k+\mu_{i}-2} \mathbf{B}, \\
& =\mathbf{0}+\alpha_{i k j} \mathbf{h}_{j} \mathbf{A}^{k^{\prime}} \mathbf{B},\left(k^{\prime}<\mu_{j}-2\right), \\
& =0
\end{aligned}
$$

It then follows that, $\overline{\mathbf{h}}_{i} \mathbf{A}^{r} \mathbf{B}=\mathbf{0}$ for $r<\mu_{i}-1$, and therefore

$$
\begin{aligned}
\overline{\mathbf{h}}_{i} \mathbf{A}^{\mu_{i}-1} \mathbf{B} & =\mathbf{h}_{i} \mathbf{A}^{\mu_{i}-1} \mathbf{B}+\alpha_{i k} \mathbf{h}_{j} \mathbf{A}^{k+\mu_{i}-1} \mathbf{B}, \\
& =\mathbf{B}_{i}^{*}+\alpha_{i k j} \mathbf{h}_{j} \mathbf{A}^{k^{*}} \mathbf{B},\left(k^{\prime \prime}<\mu_{j}-1\right), \\
& =\mathbf{B}_{i}^{*} .
\end{aligned}
$$

By considering for $\bar{h}$ in the form of Eq. (43) with all $j$ 's such that $\mu_{j}>\mu_{i}$, following the above discussion, then Eq. (44) is proved. q.e.d. 


\title{
線性系統極點安置參數化之研究
}

\author{
莊 政 義
}

國立台管海洋大受商船受系

摘 要

本文提出創新方法以最少有效自由多数作状 態回授式極點安置之参数化設部。所依理論為虚回 路解合法。针對線性可控制系統, 本文揭露出增益 矩陣所含之線性及非線性参数化影函式。在提供参 数化過程中，控制類族係由控制指数之集合指定 之, 同時做不同的回路極點指定亦可使得增益矩陣 有不同的選擇。本文之優點將以数個数值寅例做闌 释。 